\title{
Association of circular RNAs and environmental risk factors with coronary heart disease
}

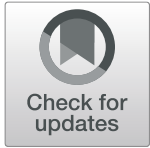

Yi Sun ${ }^{1 \dagger}$, Rong Chen ${ }^{1 \dagger}$, Shaowei Lin ${ }^{1 \dagger}$, Xiaoxu Xie ${ }^{1,2}$, Hailing Ye ${ }^{1}$, Fuli Zheng ${ }^{3}$, Jie Lin ${ }^{1}$, Qing Huang ${ }^{1}$, Shuna Huang ${ }^{1}$, Qishuang Ruan ${ }^{4}$, Tingxing Zhang ${ }^{5^{*}}$, Huangyuan $\mathrm{Li}^{3^{*}}$ and Siying $\mathrm{Wu}^{1^{*}}$ (D)

\begin{abstract}
Background: Coronary heart disease (CHD) is a complex disease caused by multi-factors and a major threat to human health. Circular RNAs (circRNAs) have critical roles in various biological processes and diseases. This study explores the independent role of circRNAs and their interaction with environmental factors in CHD.

Methods: A case-control study was conducted from March 2015 to September 2017 in Fuzhou, China. A total of 585 CHD patients and 585 gender- and age-matched healthy controls were enrolled. Questionnaire survey, health examination and molecular biology laboratory testing were conducted. Microarray technology and quantitative real-time polymerase chain reaction (PCR) were used to profile the expression levels of circRNAs. The area under the curve (AUC) of the receiver operating characteristic (ROC) was used to determine the diagnostic cut-offs. Multivariate logistic regression and multiplicative analysis were used to analyse the effects of environmental factors and hsa_circ_0008507, hsa_circ_0001946, hsa_circ_0000284 and hsa_circ_0125589 on CHD.

Results: The expression profile of circRNAs showed that 3423 circRNAs were differentially expressed at $P<0.05$, but none pass multiple testing correction. qRT-PCR further confirmed the expression levels of hsa_circ_0008507, hsa_circ 0001946 and hsa_circ_0000284 in peripheral blood leukocytes in CHD cases were higher than those in non-CHD subjects (All $p<0.05)$. Hsa_circ_0008507 (OR=1.29; 95\% Cl: 1.11-1.50), hsa_circ_0001946 (OR=1.20; 95\% Cl: 1.01-1.42) and hsa_circ_0000284 (OR=2.05; 95\% Cl: 1.32-3.19) were independent risk factors for CHD after controlling other common environmental risk factors. The AUC for hsa_circ_0008507, hsa_circ_0001946 and hsa_circ_0000284 was 0.75, 0.71 and 0.68 , respectively. Compared with non-smoking individuals with low hsa_circ_0008507 expression, the smokers with high hsa_circ_0008507 expression showed the highest magnitude of OR in CHD risk. Additionally, a statistically significant multiplicative interaction was found between hsa_circ_0008507 and smoking for CHD.

(Continued on next page)
\end{abstract}

\footnotetext{
*Correspondence: 907683161@qq.coom; fmulhy@163.com; fmuwsy@163.com

${ }^{\dagger}$ Yi Sun, Rong Chen and Shaowei Lin contributed equally to this work. ${ }^{5}$ Department of Cardiology, The First Affiliated Hospital of Fujian Medical University, Fuzhou, Fujian, China

${ }^{3}$ Department of Preventive Medicine, School of Public Health, Fujian Medical

University, Minhou County, Fuzhou, Fujian, China

${ }^{1}$ Department of Epidemiology and Health Statistics, School of Public Health,

Fujian Medical University, Minhou County, Fuzhou, Fujian, China

Full list of author information is available at the end of the article
}

(c) The Author(s). 2019 Open Access This article is distributed under the terms of the Creative Commons Attribution 4.0 International License (http://creativecommons.org/licenses/by/4.0/), which permits unrestricted use, distribution, and reproduction in any medium, provided you give appropriate credit to the original author(s) and the source, provide a link to the Creative Commons license, and indicate if changes were made. The Creative Commons Public Domain Dedication waiver (http://creativecommons.org/publicdomain/zero/1.0/) applies to the data made available in this article, unless otherwise stated. 
(Continued from previous page)

Conclusions: Hsa_circ_0008507, hsa_circ_0001946 and hsa_circ_0000284 were closely related to the occurrence and development of CHD. The combination of smoking and high hsa_circ_0008507 expression causes the occurrence and development of CHD.

Keywords: Coronary heart disease, Circular RNA, Environmental risk factors, Microarray, Interaction

\section{Background}

Coronary heart disease (CHD) is a leading global cause of death with annually increasing mortality and morbidity $[1,2]$. Family history of CHD, heavy drinking, arterial hypertension, smoking, obesity, diabetes mellitus, hypertension and physical activity are classic risk factors for CHD [3-5]. CHD is also a polygenic hereditary disease [6]. Epigenetics are called "bridges" that connect environmental factors and the genomes affecting gene expression in the absence of genomic variation, thereby affecting the sensitivity of the disease [7-9].

Circular RNAs (circRNAs) are currently noticeable in the field of RNA. They form covalently fused loops, where the RNA's $5^{\prime}$ end fuses to its $3^{\prime}$ end and removes the 5' Cap and poly(A) tail [10]. Recent studies suggested that circRNAs are widespread, abundant, conserved and tissuespecific endogenous ncRNAs in mammalian cells $[11,12]$. CircRNAs regulate gene expression by acting as miRNA sponges, RNA-binding protein sequestering agents or nuclear transcriptional regulators $[13,14]$. Despite the important role of circRNAs in the development of CHD, their relative contributions and interaction with environmental factors to CHD remain largely unknown.

In this study, $585 \mathrm{CHD}$ patients and 585 healthy controls were enrolled to evaluate the effects of environmental factors on CHD risk. Microarray and experiments involving these populations were conducted to explore the roles of dysregulated circRNAs in the CHD cases. circRNA have been tested in tissues, serum, exosomes and other body fluids in various diseases $[15$, 16]. In this study, we also validated the differentially expressed circRNAs in whole blood and peripheral blood leukocyte samples to further clarify our results. This study aims to explore the independent role of circRNAs and their interaction effect with environmental factors in the CHD.

\section{Methods}

\section{Study population}

A frequency-matched case-control study was conducted in which case groups and controls must have the same gender and age ratio $( \pm 3$ years). A research factor with a small OR was selected to calculate the appropriate sample size, according to the pre-experimental results and considered the following: $\alpha=0.05, \beta=0.10, P_{0}=0.260$ and $\mathrm{OR}=1.70$. Calculations using PASS revealed $\mathrm{N}_{1}=$
$\mathrm{N}_{2}=379$ people. In this study, 1300 questionnaires, including 650 cases and 650 controls, were distributed, and all were collected between March 2016 and September 2017 from the First Affiliated Hospital of Fujian Medical University and the Affiliated Union Hospital of Fujian Medical University, China. After corresponding the age and gender, $585 \mathrm{CHD}$ patients and 585 controls were enrolled in this study. CHD was defined using the following criteria: (1) significant stenosis ( $\geq$ $50 \%$ ) of $>1$ major coronary artery was confirmed by present cardiac catheterisation, (2) documented history of prior myocardial infarction (MI) and a prior coronary revascularisation procedure (percutaneous coronary intervention or coronary artery bypass graft), (3) patients in the stable stage after acute MI and patients with ST-segment elevation/depression on ECG. The subjects without medical history of cardiovascular diseases were selected as the non-CHD subjects. All subjects were long-term residents in Fujian, subjects with other types of heart disease, serious brain organic diseases, malignant tumours, hepatic or renal dysfunction, recent infections and endocrine system diseases were excluded from the study. This study protocol conformed to the ethical guidelines of the Declaration of Helsinki. The protocol was approved by the ethics committee of Fujian Medical University School. All selected patients and controls provided informed consent.

\section{Questionnaire survey}

All interviewer who conducted the standard questionnaire were specially trained. The questionnaire included demographic characteristics (age, gender, marital status, educational level), lifestyle habits (smoking, alcohol drinking, diet, exercise), social psychological factors (, anxiety and depression levels), Physiological index (height, weight, waist circumference (WC)) and family history of cardiovascular disease. The questionnaire was listed in Additional file 1: Table S1. Individuals who have consumed more than 20 packs of cigarettes or one cigarette a day for at least 1 year were defined as smokers. Alcohol drinkers were defined according to literature [17]. Each exercise lasting for no less than 20 min will be recorded as an effective physical exercise, according to this standard, the weekly physical activity of all subjects were determined. Anxiety and depression 
were evaluated by self-rating depression scale (SDS) and self-rating anxiety scale (SAS) [18]. SAS scores were divided into $<50,50-59,60-69$, and $\geq 70$, which were used to indicate normal, mild anxiety, moderate anxiety, and severe anxiety, respectively. SDS was categorised as $<53$, $53-62,63-73$ and $\geq 74$, which were used to indicate normal, mild depression, moderate depression, and severe depression, respectively. Body mass index $(\mathrm{BMI})=$ weight (kg) / [height (m) $]^{2}$ and categorised to three scales as follows: $<18.5 \mathrm{~kg} / \mathrm{m}^{2}$ underweight, $18.5-24.0 \mathrm{~kg} / \mathrm{m}^{2}$ normal and $\geq 24.0 \mathrm{~kg} / \mathrm{m}^{2}$ overweight and obese. A male waistline cutoff of 85 or higher means abdominal obesity, and a female waistline cutoff of 80 or more means abdominal obesity. According to gender, WC was classified into two categories: A male WC with a cutoff of 85 or higher implies abdominal obesity, whereas a female WC with a cut-off value of 80 or more suggests abdominal obesity.

\section{CircRNA microarray analysis}

The samples used for microarray analysis include 4-12 cases [19]. Therefore, five patients with similar age, disease and disease duration and with no other diseases and five controls with similar general conditions, age and sex was selected for microarray analysis. The distribution of demographic variables is listed in Additional file 2: Table S2. The RNAs of the peripheral blood samples from five CHD cases and five non-CHD subjects were extracted for microarray analysis. According to the manufacturer's instructions, total RNA was extracted from $1 \mathrm{~mL}$ of whole blood sample by using a fast total RNA extraction kit (Bioteke, Beijing, China). RNA was then dissolved in RNase-free water. NanoDrop1000 instrument (NanoDrop, England) was used to measure the yield and purity of the RNA.. All total RNA was of high purity with OD260 / 280 in the range of 1.80-2.00. 1\% formaldehyde denaturing gel electrophoresis was used to determine the integrity of the RNA. The extracted RNAs were digested, dephosphorylated, denatured, amplified and labelled with Cy3-dCTP according to the manufacturer's specifications. The purified RNAs were hybridised to a microarray (Agilent human circRNA Array V2.0) containing 170,340 human circRNA probes. GeneSpring software V13.0 (Agilent Technologies, Santa Clara, CA, USA) was then used to analyze microarray data for circRNA. The thresholds were fold change (FC) of $>2$ or $<-2$ and $p<0.05$ according to the $\mathrm{t}$-test. The data were $\log -2$ transformed and median-centred by genes through CLUSTER 3.0 software and then analysed using hierarchical clustering with average linkage.

\section{RNA extraction}

Representative subjects were selected using stratified sampling from the first part of the study. The subjects in the case group were divided into two layers according to age (i.e. $<65$ years old and $\geq 65$ years old) and stratified by gender according to each age group. The sex ratio of all subjects in the case group were determined. According to the random number table, the corresponding number of male and female subjects was finally selected at each age level. The control group were also sampled using the same method. According to the pre-experimental results, the PASS software obtained the following result: $\mathrm{N}_{1}=\mathrm{N}_{2}=25$, considering $\alpha=0.05, \beta=0.10, \mathrm{P}_{0}=0.50$ and $\mathrm{OR}=1.8$. Therefore, at least 25 samples were required in each group. Therefore, 30 cases and controls were finally determined for peripheral blood quantitative real-time polymerase chain reaction (qRT-PCR) validation, and 100 cases and controls were used for peripheral blood leukocyte qRT-PCR validation. A fast total RNA extraction kit (Bioteke, Beijing, China) was used to extract total RNA from peripheral blood in 30 CHD patients and 30 non-CHD controls. Total RNA from peripheral blood leukocytes in $100 \mathrm{CHD}$ patients and 100 non-CHD controls was extracted by using TRIzol reagent (Invitrogen, USA). The examination of RNA concentration, purity, and integrity were as described above.

\section{cDNA synthesis and qRT-PCR}

Reverse transcription of quantified RNA was performed using PrimeScript RT Reagent Kit (Takara Bio Inc., Shiga, Japan) according to the manufacturer's instructions. qRT-PCR was used to measure the expression levels of circRNAs, Which was performed on the LightCycler 480 Real-Time PCR System (Roche, Switzerland) with the SYBR ${ }^{\circ}$ Premix Ex Taq $^{\text {mi }}$ II kit (Takara Bio Inc., Shiga, Japan). The reaction conditions were listed as follows: Amplification curves were obtained by 45 cycles of

Table 1 Baseline characteristics of study subjects

\begin{tabular}{lllll}
\hline Characteristics & $\mathrm{CHD}, \mathrm{n}(\%)$ & Non-CHD, $\mathrm{n}(\%)$ & $x^{2}$ & $p$-value \\
\hline Gender & & & 0.000 & 1.000 \\
$\quad$ Male & $318(54.4)$ & $318(54.4)$ & & \\
$\quad$ Female & $267(45.6)$ & $267(45.6)$ & & \\
Age & & & 0.777 & 0.378 \\
$<65$ & $271(46.3)$ & $256(43.8)$ & & \\
$\geq 65$ & $314(53.7)$ & $329(56.2)$ & & \\
Marital status & & & 0.380 & 0.538 \\
$\quad \begin{array}{l}\text { Marriage } \\
\text { Single and others }\end{array}$ & $49(8.4)$ & $55(9.4)$ & & \\
Education level & & & 1.108 & 0.575 \\
Below primary school & $260(44.4)$ & $263(45.0)$ & & \\
Middle school & $257(43.9)$ & $265(45.3)$ & & \\
College or higher & $68(11.6)$ & $57(9.7)$ & & \\
\hline
\end{tabular}

CHD coronary heart disease, Non-CHD None coronary heart disease 
$95^{\circ} \mathrm{C} 30 \mathrm{~s}, 95^{\circ} \mathrm{C} 5 \mathrm{~s}, 60^{\circ} \mathrm{C} 34 \mathrm{~s}$, whereas dissolution curves were obtained by one cycle of $95^{\circ} \mathrm{C} 15 \mathrm{~s}, 60^{\circ} \mathrm{C} 1$ min, $95^{\circ} \mathrm{C} 15 \mathrm{~s}$. GAPDH was used as internal control. $2^{-\triangle \Delta C T}$ method was used to calculate the expression levels of circRNAs. The primer sequences are shown in Additional file 3: Table S3.

\section{Statistical analyses}

Data with normal distribution were presented as the mean value \pm SD and compared using two-tailed Student's t-test. Skewed data were represented as median (25th-75th quartile) and compared using Mann-Whitney $U$ test. Discrete variables were displayed as percentages, distribution differences were examined by the chisquare $\left(x^{2}\right)$ test. The diagnostic cut-offs of circRNAs were obtained from the receiver operating characteristic
(ROC) curve. Univariate analysis of meaningful variables was included in the multivariate analysis. Crossover analysis was used to assess the association between CHD and risk factors. A $p$-value $<0.05$ (two-tailed) was considered significant. All statistical analyses were performed using SPSS 25.0 software.

\section{Results}

\section{Baseline demographic characteristics}

The distribution of all subjects according to demographic variables is shown in Table 1 . No significant difference was found in their general demographic characteristics, including age, gender, marital status and educational level (all $p>0.05$ ), thereby indicating that the frequency matching was adequate (Table 1).

Table 2 Univariate and Multiple logistic regression analysis of environmental factors and CHD risk

\begin{tabular}{|c|c|c|c|c|}
\hline \multirow[t]{2}{*}{ Factors } & \multicolumn{2}{|c|}{ Univariate logistic regression } & \multicolumn{2}{|c|}{ Multivariate logistic regression } \\
\hline & OR $(95 \% \mathrm{Cl})$ & $p$-value & $\mathrm{OR}^{\mathrm{a}}(95 \% \mathrm{Cl})$ & $p$-value \\
\hline Smoking & $1.06(0.83-1.35)$ & 0.661 & - & - \\
\hline Passive smoking & $1.08(0.82-1.43)$ & 0.569 & - & - \\
\hline Alcohol drinking & $1.06(0.76-1.46)$ & 0.741 & - & - \\
\hline High-salt diets & $1.75(1.35-2.28)$ & $<0.001$ & $1.20(0.76-1.88)$ & 0.438 \\
\hline Light diets & $0.60(0.47-0.76)$ & $<0.001$ & $0.78(0.51-1.20)$ & 0.260 \\
\hline \multicolumn{5}{|l|}{ Physical exercise } \\
\hline$<1$ time/week & 1.00 & - & 1.00 & - \\
\hline 1-2 times/week & $0.45(0.25-0.80)$ & 0.007 & $0.41(0.23-0.75)$ & 0.004 \\
\hline$\geq 3$ times/week & $0.96(0.75-1.22)$ & 0.713 & $0.94(0.73-1.21)$ & 0.608 \\
\hline \multicolumn{5}{|l|}{ Character } \\
\hline B type & 1.00 & - & 1.00 & - \\
\hline A type & $1.29(0.99-1.69)$ & 0.060 & - & - \\
\hline C type & $1.43(0.93-2.17)$ & 0.105 & - & - \\
\hline D type & $1.56(0.68-3.55)$ & 0.294 & - & - \\
\hline Anxiety & $2.32(1.69-3.19)$ & $<0.001$ & $2.34(1.67-3.28)$ & $<0.001$ \\
\hline \multicolumn{5}{|l|}{ Depression } \\
\hline Normal & 1.00 & - & 1.00 & - \\
\hline Mild & $1.33(0.93-1.90)$ & 0.120 & - & - \\
\hline Moderate/Severe & $1.40(0.84-2.35)$ & 0.196 & - & - \\
\hline \multicolumn{5}{|l|}{ BMI } \\
\hline $18.50-23.99$ & 1.00 & - & 1.00 & - \\
\hline$<18.50$ & $0.69(0.39-1.21)$ & 0.192 & $0.68(0.37-1.22)$ & 0.194 \\
\hline$\geq 24.00$ & $1.50(1.18-1.90)$ & 0.001 & $1.47(1.13-1.91)$ & 0.004 \\
\hline Abdominal obesity & $1.41(1.08-1.86)$ & 0.013 & $1.11(0.81-1.51)$ & 0.530 \\
\hline Family history of hypertension & $1.16(0.91-1.47)$ & 0.228 & - & - \\
\hline Family history of stroke & $1.44(0.92-2.25)$ & 0.114 & - & - \\
\hline Family history of diabetes & $1.14(0.82-1.59)$ & 0.445 & - & - \\
\hline
\end{tabular}

$O R$ odds ratio, $\mathrm{Cl}$ confidence interval

${ }^{a}$ Data are adjusted for age, gender, marital status, and education level, Statistically significant variables in univariate analysis were selected for further multivariate analysis 


\section{Environmental factors and CHD}

Univariate analysis on the associations between individual behavioural factors and risk of CHD showed that salty diet. Social psychological factor analysis showed that anxious people are highly prone to CHD. BMI $(\geq 24.00)$ and abdominal obesity were significantly different in CHD and non-CHD subjects. However, light diets and physical exercise (one to two times per week) were protected factors for CHD. Unconditional logistic regression analysis was further used to evaluate the associations between the environmental factors (high-salt diet, light diet, active exercise, anxiety, BMI and abdominal obesity) and CHD. Significant increased risk effects for CHD were associated with anxiety (odds ratio $(\mathrm{OR})=2.34$; 95\% confidence interval (95\% CI): $1.67-3.28\}$ and being overweight $(\mathrm{BMI} \geq 24.00) \quad\{\mathrm{OR}=1.47 ; 95 \% \mathrm{CI}$ : 1.13-1.91\}. Physical exercise (one to two times per week) $\{\mathrm{OR}=0.41 ; 95 \% \mathrm{CI}: 0.23-0.75\}$ was the protective factor for CHD (Table 2).

Identification of dysregulated circRNA expression profiles The heat map, volcano plot and scatter plot of microarray assay showed abnormal expression of circRNAs in the CHD cases (Fig. 1). The microarray study identified that 3423 circRNAs were differentially expressed in
$\mathrm{CHD}$ and non-CHD subjects based on FC and $p$-value $(\mathrm{FC}>2, p<0.05)$. Four circRNAs were selected for qRTPCR validation analysis to independently validate our results and determine the roles of circRNAs in CHD. Selection was based on the following: (1) hsa_circ 0008507, hsa_circ_0125589 and hsa_circ_0000284 are among the most abundant and have significantly differentially expression according to microarray analysis, (2) overexpression of hsa_circ_0001946 in cardiac myocyte cells promotes cell apoptosis [20] and silencing hsa circ_0000284 can alleviate retinal vascular dysfunction [21]. In summary, the circRNAs associated with the occurrence and development of CHD include hsa_circ 0125589, hsa_circ_0008507, hsa_circ_0001946 and hsa_ circ_0000284. (Table 3).

\section{Expression of circRNAs in peripheral blood}

We selected $30 \mathrm{CHD}$ cases and 30 non-CHD controls with corresponding age and gender as qRT-PCR subjects. The expression levels of hsa_circ_0001946 and hsa_circ_0000284 in CHD were significantly elevated compared with those in the non-CHD subjects (Fig. 2). No significant differences were found in the expression levels of hsa_circ_0125589 and hsa_circ_0008507. The expression level of hsa_circ_0001946 was consistent with

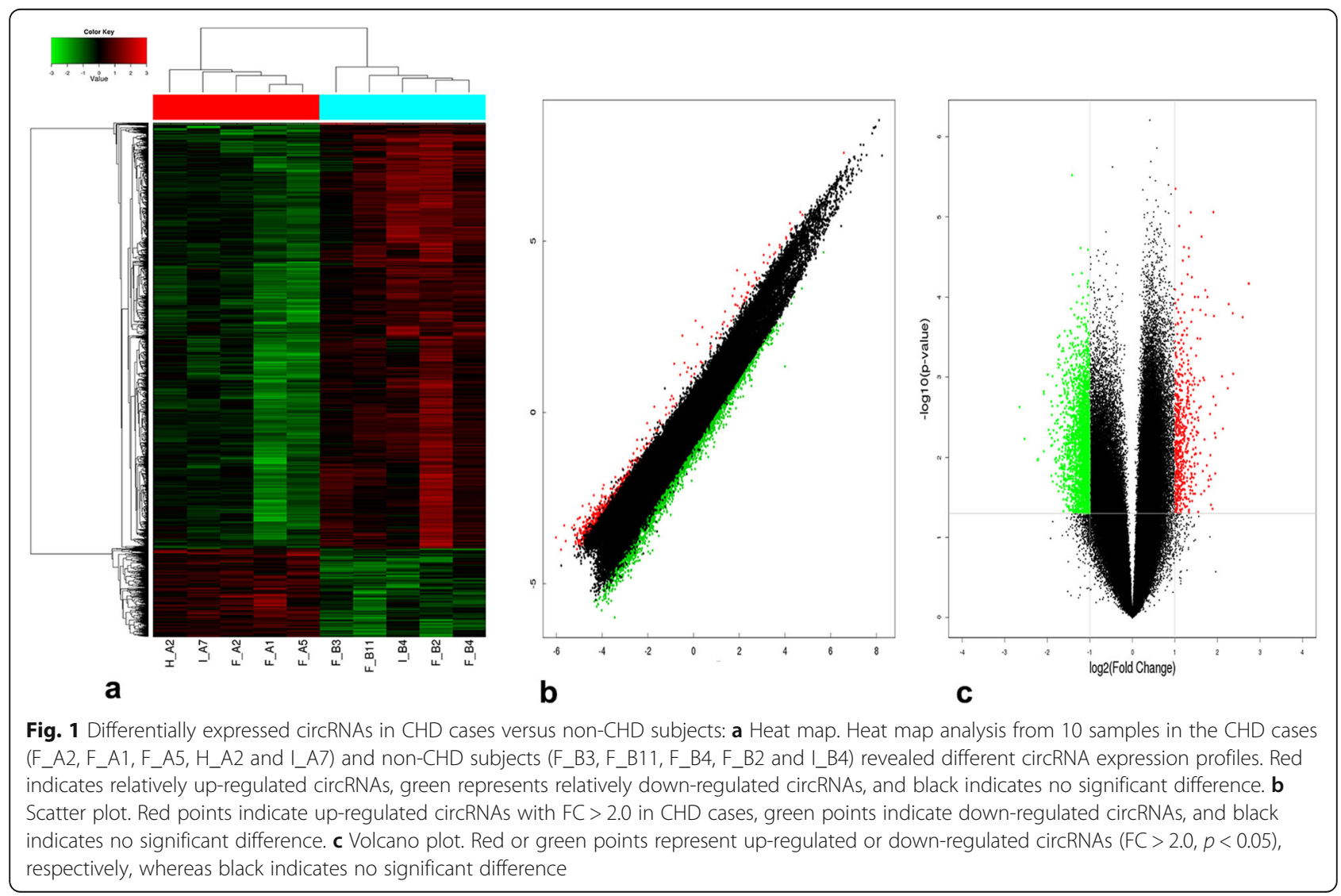


Table 3 The basic information of 4 selected circRNAs

\begin{tabular}{llll}
\hline CircRNA ID & FC & $p$-value & Regulation \\
\hline hsa_circ_0125589 & 4.64 & 0.010 & Down \\
hsa_circ_0008507 & 4.31 & $<0.001$ & Up \\
hsa_circ_0001946 & 1.39 & 0.088 & Up \\
hsa_circ_0000284 & 2.24 & 0.008 & Down \\
\hline
\end{tabular}

FC fold change, circRNA Circular RNA

the microarray assay, whereas that of hsa_circ_0000284 was contrary to the result of the microarray.

\section{Validation of dysregulated circRNAs of peripheral blood leukocytes by qRT-PCR}

We selected 100 CHD cases and 100 non-CHD controls as qRT-PCR subjects, all of which corresponded in terms of age and gender. The expression levels of hsa_circ 0001946 and hsa_circ_0000284 in the peripheral blood leukocytes in CHD group were significantly elevated compared with those in the non-CHD subjects. No significant difference was found in the expression level of hsa_circ_0125589 in peripheral blood leukocytes between the case and control groups $(p>0.05)$ (Fig. 3). These results were consistent with the qRT-PCR validation of peripheral blood samples. However, the expression level of hsa_circ_0008507 was up-regulated, which was inconsistent with the qRT-PCR validation of peripheral blood samples. The expression levels of hsa circ_0008507 and hsa_circ_0001946 were consistent with the microarray assay, whereas that of hsa_circ 0000284 was contrary to the result of the microarray assay.

\section{Multivariate analysis of circRNAs and environmental factors}

Two logistic regression analysis models were further used to analyse the association of factors (age, gender, marital status and education level in Model 1 and age, gender, marital status, education level, anxiety, BMI and physical activity in Model 2) with circRNA. CHD was the dependent variable $(0=$ no, $1=$ yes $)$, and the related environmental factors and four verified circRNAs in peripheral blood leukocytes were the independent variables. The results suggested that the high expression levels of hsa_circ $0008507\{(\mathrm{OR}=1.30 ; 95 \% \mathrm{CI}: 1.13-1.51$ in Model 1$)$, $(\mathrm{OR}=1.29$; $95 \% \mathrm{CI}: 1.11-1.50$ in Model 2)\}, hsa_circ $0001946\{(\mathrm{OR}=1.25 ; 95 \% \mathrm{CI}: 1.06-1.47$ in Model 1$)$, $(\mathrm{OR}=1.20$; $95 \% \mathrm{CI}: 1.01-1.42$ in Model 2) , hsa_circ_ $0000284\{(\mathrm{OR}=1.88 ; 95 \% \mathrm{CI}: 1.24-2.84$ in Model 1$)$,
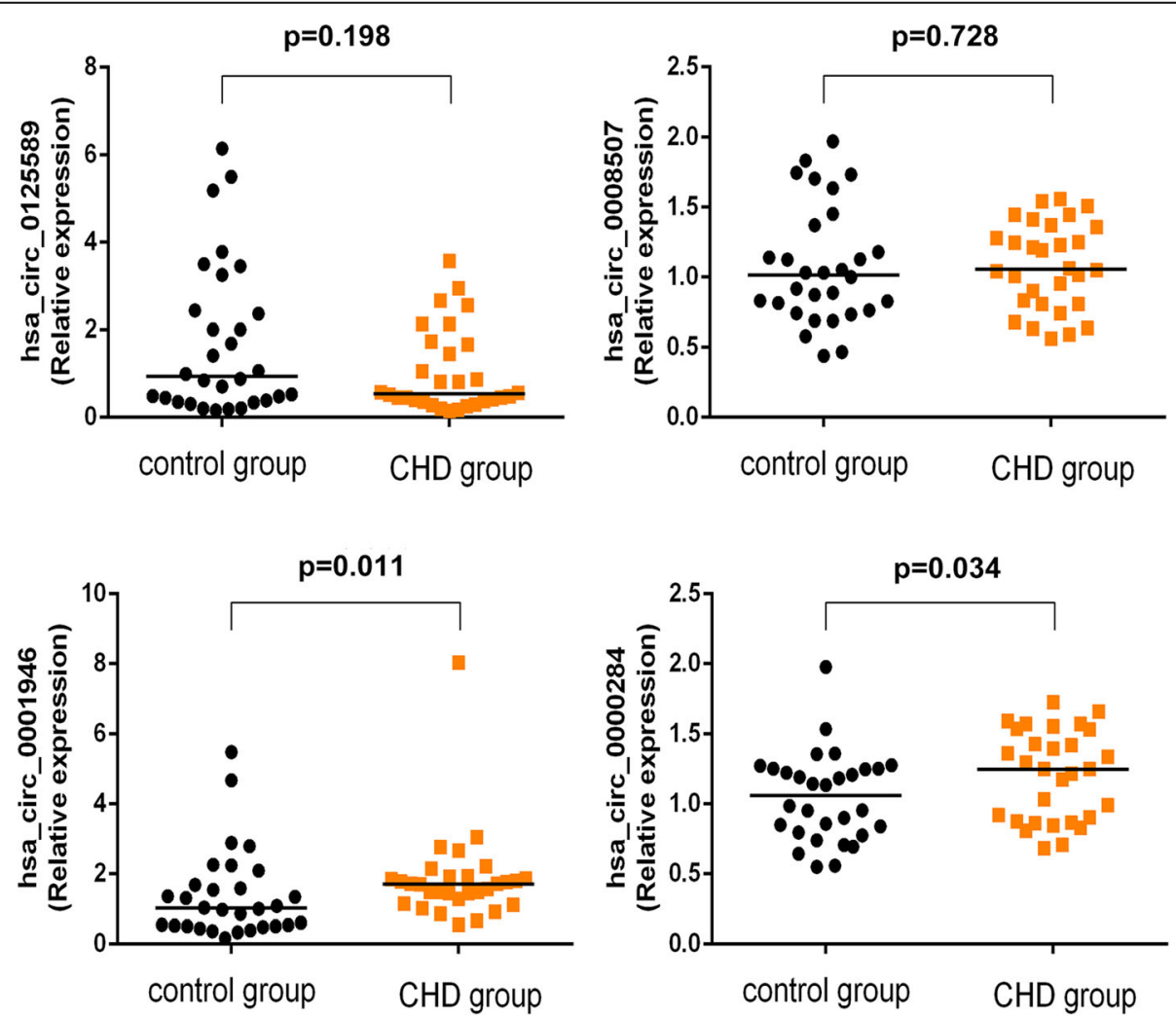

Fig. 2 Comparison of the expression levels of circRNAs between CHD and control subjects: four differentially expressed circRNAs were validated by qRT-PCR. Data are expressed as the median (25th-75th quartile) 

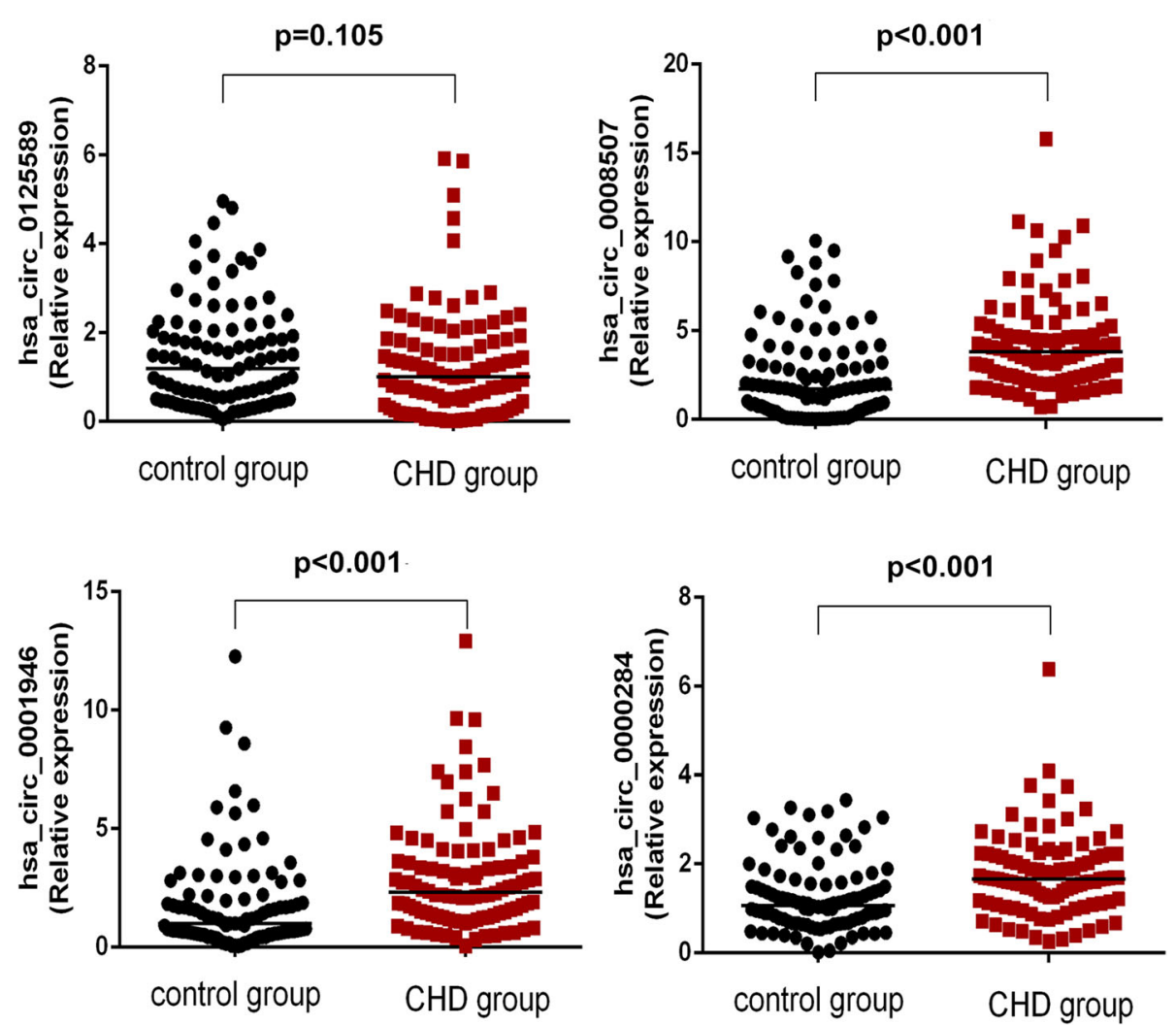

Fig. 3 Comparison of the expression levels of circRNAs between CHD and non-CHD subjects of peripheral blood leukocytes: Four differentially expressed circRNAs were validated by qRT-PCR. Data are expressed as the median (25th-75th quartile)

$(\mathrm{OR}=2.05$; 95\% CI: $1.32-3.19$ in Model 2) $\}$ were the independent risk factors of CHD in the two models (Table 4).

\section{Determining diagnostic cut-offs by ROC curve}

The ROC curve in 100 cases and 100 controls of peripheral blood leucocytes of hsa_circ_0008507 showed an AUC of 0.75 (95\% CI: 0.68-0.82). The maximum Youden's index was 0.46 with a sensitivity of 0.86 and a specificity of 0.60 , corresponding to a diagnostic cut-off of 1.975. The ROC curve of hsa circ_0001946 showed that the AUC was 0.71 (95\% CI: 0.64-0.79). The maximum Youden's index was

Table 4 Multivariable logistic regression analyses of the verified circRNAs and environmental factors in CHD risk

\begin{tabular}{llllll}
\hline Factors & Model 1 & & & Model 2 & \\
\cline { 2 - 3 } \cline { 6 - 7 } & OR $(95 \% \mathrm{Cl})$ & $p$-value & & OR $(95 \% \mathrm{Cl})$ & $p$-value \\
\hline hsa_circ_0008507 & $1.30(1.13-1.51)$ & $<0.001$ & & $1.29(1.11-1.50)$ & 0.001 \\
hsa_circ_0001946 & $1.25(1.06-1.47)$ & 0.007 & & $1.20(1.01-1.42)$ & 0.033 \\
hsa_circ_0000284 & $1.88(1.24-2.84)$ & 0.003 & & $2.05(1.32-3.19)$ & 0.002 \\
hsa_circ_0125589 & $0.97(0.73-1.29)$ & 0.821 & & $0.960(0.71-1.29)$ & 0.779
\end{tabular}

Model 1: Adjusted for age, gender, marital status, and education level Model 2: Adjusted for age, gender, marital status, education level, anxiety, BMI and physical activity

$O R$ odds ratio, $\mathrm{Cl}$ confidence interval, $\mathrm{BMI}$ Body mass index
0.37 with a sensitivity of 0.85 and a specificity of 0.52 , corresponding to a diagnostic cut-off of 1.00 . The ROC curve of hsa_circ_0000284 showed that the AUC was 0.68 (95\% CI: 0.61-0.76). The maximum Youden's index was 0.37 with a sensitivity of 0.66 and a specificity of 0.71 , corresponding to a diagnostic cut-off of 1.42 (Fig. 4).

\section{Combined analysis of environmental factors and epigenetic factors in CHD}

The diagnostic cut-offs were used to define high and low expression groups of hsa_circ_0008507, hsa_circ_0001946 and hsa_circ_0000284. Stratification by demographic characteristics and main lifestyle factors revealed the statistically significant associations between hsa_circ_0008507, hsa circ_0001946 and CHD risk in young and old individuals, males and females, smokers and non-smokers, abdominal obesity and non-abdominal obesity individuals and passive smokers and active smokers. The statistically significant associations between hsa_circ_0000284 and CHD risk were also observed in these comparison groups. In addition, the combined effect of hsa_circ_0008507 and smoking was observed. Compared with non-smoking individuals with low hsa_circ_0008507 expression, smokers with high hsa_circ 0008507 expression showed the highest magnitude of OR 


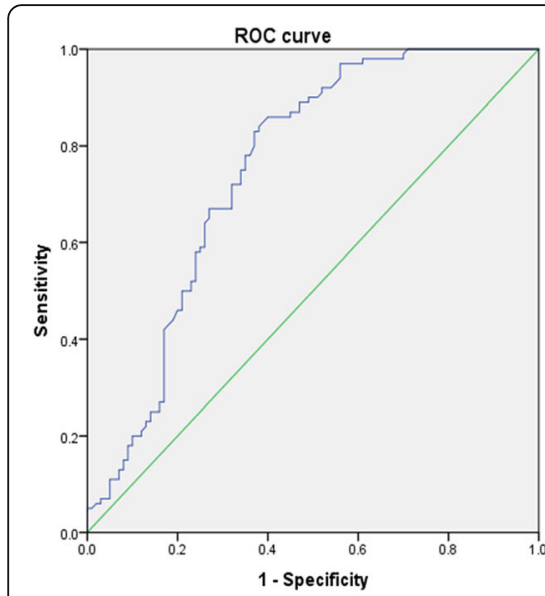

a

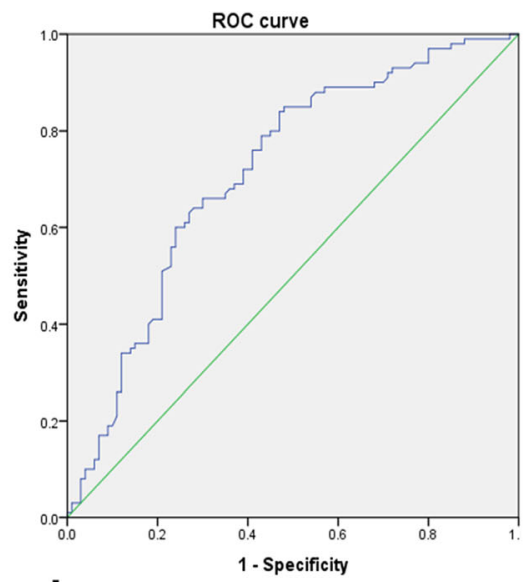

b

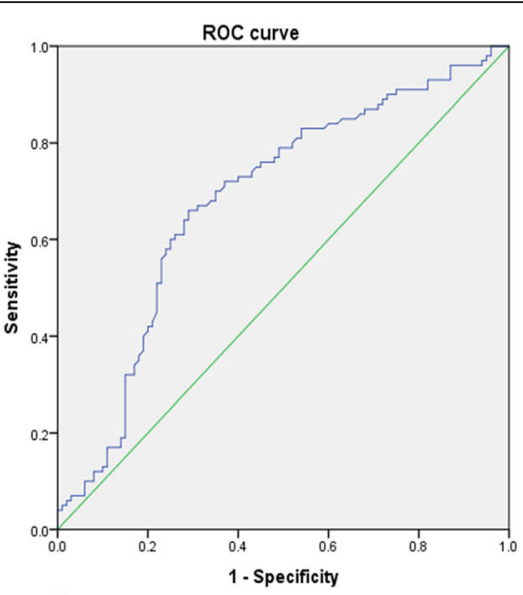

C

Fig. 4 circRNAs in 200 cases of peripheral blood leukocytes in the diagnosis of CHD ROC curve. a ROC curve for hsa_circ_0008507; b ROC curve for hsa_circ_0001946; c ROC curve for hsa_circ_0000284

in CHD risk (OR 6.94, 95\% CI 1.95-24.72). A statistically significant multiplicative interaction was found between hsa_circ_0008507 and smoking for CHD (OR multiplicative: 5.68; 95\% CI: 1.08-29.84; $p=0.040$ ) (Tables 5 and 6).

\section{Discussion}

We confirmed that high BMI and hsa_circ_0008507, hsa circ_0001946, hsa_circ_0000284 expression levels are associated with CHD, thereby indicating that CHD is a complex disease affected by multiple levels of factors. Furthermore, combined analysis results show that smoking combined with hsa_circ_0008507 induces the development of CHD.
Among the common risk factors, high BMI is associated with a significantly increased risk of CHD, and this finding is consistent with the report of Fritz J et al. and Y Chen et al. [22, 23]. Increased body mass accompanied by highpowered circulation, chronic volume overload and increased preload and afterload caused by increased peripheral resistance is a risk factor for cardiovascular disease [24].

The expression levels of hsa_circ_0001946, hsa_circ_ 0008507 and hsa_circ_0000284 were significantly elevated in CHD peripheral blood leukocytes. The overexpression of Hsa_circ_0001946 (also called Cdr1as) in cardiac myocyte cells promotes cell apoptosis [20]. In the present

Table 5 Risk odds ratios of CHD for circRNAs stratified by selected characteristics

\begin{tabular}{|c|c|c|c|c|c|c|}
\hline \multirow[b]{2}{*}{ Variable } & \multicolumn{2}{|l|}{ hsa_circ_0008507 } & \multicolumn{2}{|l|}{ hsa_circ_0001946 } & \multicolumn{2}{|l|}{ hsa_circ_0000284 } \\
\hline & Adjusted OR(95\% Cl) & Pinteraction & Adjusted OR(95\% Cl) & $\mathrm{p}_{\text {interaction }}$ & Adjusted OR(95\% Cl) & $\mathrm{P}_{\text {interaction }}$ \\
\hline \multicolumn{7}{|l|}{ Age } \\
\hline$<65$ & $16.82(4.15-68.20)$ & 0.808 & $6.75(1.86-24.43)$ & 0.865 & $5.39(1.91-15.22)$ & 0.920 \\
\hline$\geq 65$ & $9.70(3.46-27.15)$ & & $6.54(2.45-17.47)$ & & $7.18(2.62-19.70)$ & \\
\hline \multicolumn{7}{|l|}{ Gender } \\
\hline Male & $19.18(5.65-65.09)$ & 0.065 & $8.89(2.90-26.66)$ & 0.371 & $3.47(1.35-8.93)$ & 0.169 \\
\hline Female & $4.88(1.74-13.67)$ & & $3.48(1.14-10.61)$ & & $11.17(3.44-36.33)$ & \\
\hline \multicolumn{7}{|l|}{ Smoking } \\
\hline No & $4.82(1.96-11.88)$ & 0.040 & $3.60(1.35-9.59)$ & 0.070 & $10.18(3.66-28.33)$ & 0.148 \\
\hline Yes & $25.25(5.84-109.22)$ & & $13.30(3.50-50.49)$ & & $3.12(1.010-9.63)$ & \\
\hline \multicolumn{7}{|c|}{ Abdominal obesity } \\
\hline No & $18.34(2.46-136.77)$ & 0.931 & $6.58(1.22-35.61)$ & 0.799 & 11.65 (1.72-78.94) & 0.476 \\
\hline Yes & $11.30(4.31-29.58)$ & & $7.93(2.92-21.54)$ & & $4.48(2.03-10.34)$ & \\
\hline \multicolumn{7}{|c|}{ Passive smoking } \\
\hline No & $8.78(3.57-21.61)$ & 0.747 & $4.80(2.00-11.49)$ & 0.363 & $5.17(2.31-12.49)$ & 0.969 \\
\hline Yes & $11.72(2.07-66.40)$ & & $42.52(3.28-551.24)$ & & $3.47(0.77-15.64)$ & \\
\hline
\end{tabular}

Data are adjusted for age, gender, marital status, education level, anxiety, BMI, and physical activity $O R$ odds ratio, $\mathrm{Cl}$ confidence interval 
Table 6 Joint effect of smoking and hsa_circ_0008507

\begin{tabular}{llllll}
\hline Smoking & hsa_circ_0008507 high expression & $\begin{array}{l}\text { Cases } \\
N(\%)\end{array}$ & $\begin{array}{l}\text { Controls } \\
N(\%)\end{array}$ & OR(95\% Cl) \\
\hline 0 & 0 & $11(11.0)$ & $30(30.0)$ & 1.00 & \\
0 & 1 & $54(54.0)$ & $28(28.0)$ & $4.84(1.97-11.88)$ & 0.001 \\
1 & 0 & $3(3.0)$ & $30(30.0)$ & $0.25(0.05-1.21)$ & 0.085 \\
1 & 1 & $32(32.0)$ & $12(12.0)$ & $6.94(1.95-24.72)$ & 0.003 \\
Multiplication interaction & & & & $5.68(1.08-29.84)$ & 0.040 \\
\hline
\end{tabular}

Data are adjusted for age, gender, marital status, education level, anxiety, BMI, and physical activity

study, hsa_circ_0001946 was up-regulated in CHD, and miR-7-5p was found in its target gene based on bioinformatics analysis. miR-7-5p directly inhibits epidermal growth factor receptor mRNA, further antagonises downstream protein kinases, induces apoptosis and inhibits cell proliferation, migration and invasion [25]. Therefore, we speculate that hsa_circ_0001946 may be involved in the development of vascular endothelium through the hsa circ_0001946/miR-7-5p pathway.

Hsa_circ_0000284 (also called circHIPK3) mediates retinal vascular dysfunction in diabetes mellitus [21]. Retinal endothelial cell viability and endothelial cell abnormal proliferation, migration and tube formation in vitro and alleviates retinal vascular dysfunction was reduced by silencing of circHIPK3, such as reducing vascular leakage and inflammation, as well as the number of diabeticinduced cellular capillaries in vivo. Population experimental studies showed that hsa_circ_0000284 is associated with carotid plaque rupture and stroke [26]. These results reveal that hsa_circ_0000284 is closely related to the growth, proliferation and plaque rupture of vascular endothelial cells.

The qRT-PCR results were incompletely consistent with those of the microarray analysis. The possible causes for this contradiction are as follows: (1) microarray analysis is only a tool for initial screening. It has high sensitivity and poor reliability. Therefore, the final result is based on the PCR verification result. (2) Differences in research objects: the same sample could not satisfy the requirement for microarray analysis and PCR verification at the same time due to the limited number of experimental samples. Therefore, the two groups of subjects tested on the microarray analysis and validated by PCR are different. (3) Sample size: the microarray analysis is costly; only five cases and five controls were selected, and the individual differences were not excluded.

Peripheral blood leukocytes can participate in the development of CHD through processes, such as blood vessel adhesion [27, 28]. Therefore, large-sample peripheral blood leukocyte qRT-PCR verification was further conducted. The results show that hsa_circ_0125589, hsa_circ_ 0008507, hsa_circ_0001946 and hsa_circ_0000284 are consistent in peripheral blood and peripheral blood leukocytes.

Stratified analysis shows that hsa_circ_0008507, hsa circ_0001946 and hsa_circ_0000284 exhibit significant differences in various populations. A statistically significant multiplicative interaction is found between hsa_circ_0008507 and smoking. Smoking can reduce the bioavailability of nitric oxide, further promote the expression of adhesion molecules and endothelial dysfunction and increase the adhesion of platelets and macrophages, thereby leading to coagulation and inflammation. Smoking can also promote the differentiation of macrophages into foam cells, thereby aggravating the progression of CHD [29, 30]. Therefore, the interaction between smoking and the high expression of hsa circ_0008507 results in a high risk of CHD.

This study has the following deficiencies. (1) In this case-control study, we collected the subject's exposure through a structured questionnaire. Although objective data were selected as much as possible, the possibility that the respondent's memory is distorted or incomplete still exists. Hence, information bias is inevitable. (2) The representative sample is insufficient because the cases and controls were only obtained from two hospitals in Fuzhou, indicating admission rate bias. (3) The expression level of circRNAs cannot be dynamically evaluated due to the lack of detection at multiple time points and the limitation on the causal association between circRNA and CHD.

\section{Conclusion}

Hsa_circ_0008507, hsa_circ_0001946 and hsa_circ 0000284 are closely related to the occurrence and development of CHD. In addition, the combination of smoking and high hsa_circ_0008507 expression induces the occurrence and development of CHD.

\section{Additional files}

Additional file 1: Table S1. the questionnaire used for investigation. The questionnaire included demographic characteristics (age, gender, marital status, educational level), lifestyle habits (smoking, alcohol 
drinking, diet, exercise), social psychological factors ( anxiety and depression levels), Physiological index (height, weight, waist circumference (WC)) and family history of cardiovascular disease. (DOC $62 \mathrm{~kb}$ )

Additional file 2: Table S2. Baseline characteristics of subjects used for microarray analysis. Five patients with similar age, disease and disease duration and with no other diseases and five controls with similar general conditions, age and sex was selected for microarray analysis. (DOC $37 \mathrm{~kb}$ )

Additional file 3: Table S3. List of the primers used for qRT-PCR experiments. The primers used for qRT-PCR experiments was listed in Table S3. (DOC $32 \mathrm{~kb})$

\section{Abbreviations}

AUC: Area under the curve; BMl: Body mass index; CHD: Coronary heart disease; Cl: Confidence interval; CircRNAs: Circular RNAs; OR: Odds ratio; qRTPCR: Quantitative real-time polymerase chain reaction; ROC: Receiveroperating characteristic

\section{Acknowledgements}

The authors would like to thank the doctors and patients for their support and cooperation in sample collection and questionnaire completion.

\section{Ethic approval and consent to participate}

The study was performed according to good clinical practice and in compliance with the Helsinki declaration. An individual written consent was obtained from each patient. The study was approved by the ethics committee of Fujian Medical University School (No. 23, March 10, 2017).

\section{Authors' contributions}

Designed and revised the manuscript: SYW, HYL and TXZ; conceived the study and were involved in the experimental design, coordination and drafting the manuscript: YS, RC, SWL, XXX, HLY, FLZ and JL; Data collection, result interpretation and manuscript writing: $Y S, R C, X X X, Q H, S N H$ and QSR; Analysed the data: SWL, HLY, FLZ, JL, QH, SNH and QSR. All of the authors have given final approval of the version to be published.

\section{Funding}

This study was supported by the Young and Middle-aged Talent-training Project in Fujian province health system (Grant No. 2014-ZQN-ZD-24); And the Natural Science Foundation of Fujian Province (Grant No. 2019 J01315). 2014-ZQN-ZD-24 was involved in study design, data collection, and analysis and interpretation of data. $2019 \mathrm{~J} 01315$ contributed to the review and the editing of this manuscript.

\section{Availability of data and materials}

The datasets generated and/or analysed during the current study are not publicly available due the principle of confidentiality of funding but are available from the corresponding author on reasonable request.

\section{Consent for publication}

Not applicable

\section{Competing interests}

The authors declare that they have no competing interests.

\section{Author details}

'Department of Epidemiology and Health Statistics, School of Public Health Fujian Medical University, Minhou County, Fuzhou, Fujian, China. ${ }^{2}$ National Research Institute for Health and Family Planning, Beijing, China.

${ }^{3}$ Department of Preventive Medicine, School of Public Health, Fujian Medical University, Minhou County, Fuzhou, Fujian, China. ${ }^{4}$ Department of Orthopedics, Fujian Medical University Union Hospital, Fuzhou, Fujian, China. ${ }^{5}$ Department of Cardiology, The First Affiliated Hospital of Fujian Medical University, Fuzhou, Fujian, China.
Received: 19 August 2018 Accepted: 25 August 2019

Published online: 16 October 2019

\section{References}

1. GBD 2016 Mortality Collaborators. Global, regional, and national under-5 mortality, adult mortality, age-specific mortality, and life expectancy, 19702016: a systematic analysis for the Global Burden of Disease Study 2016. Lancet. 2017;390(10100):1084-150.

2. Kilic S, Sümerkan $M C$, Emren $V$, et al. Secondary prevention of coronary heart disease in elderly population of Turkey: a subgroup analysis of ELDERTURK study. Cardiol J. 2019:26(1):13-19.

3. Li Z, Bai Y, Guo X, et al. Alcohol consumption and cardiovascular diseases in rural China. Int J Cardiol. 2016;215:257-62.

4. Z Zhang JX, Dong HZ, Chen BW, et al. Characteristics of coronary arterial lesions in patients with coronary heart disease and hypertension. Springerplus. 2016;5(1):1208.

5. Stewart RAH, Held C, Hadziosmanovic N, et al. Physical activity and mortality in patients with stable coronary heart disease. J Am Coll Cardiol. 2017; 70(14):1689-700.

6. Mack M, Gopal A. Epidemiology, traditional and novel risk factors in coronary artery disease. Cardiol Clin. 2014;32(3):323-32

7. Huan $T$, Rong J, Tanriverdi $K$, et al. Dissecting the roles of microRNAs in coronary heart disease via integrative genomic analyses. Arterioscler Thromb Vasc Biol. 2015:35(4):1011-21.

8. Turner AW, Martinuk A, Silva A, et al. Functional analysis of a novel genomewide association study signal in SMAD3 that confers protection from coronary artery disease. Arterioscler Thromb Vasc Biol. 2016;36(5):972-83.

9. Fan $X$, Weng $X$, Zhao $Y$, et al. Circular RNAs in cardiovascular disease: an overview. Biomed Res Int. 2017;2017:5135781.

10. Chen LL, Yang L. Regulation of circRNA biogenesis. RNA Biol. 2015; 12(4):381-8.

11. Rybak-Wolf A, Stottmeister C, Glažar P, et al. Circular RNAs in the mammalian brain are highly abundant, conserved,and dynamically Expressed. Mol Cell. 2015;58(5):870-85.

12. Szabo L, Morey R, Palpant NJ, et al. Statistically based splicing detection reveals neural enrichment and tissue-specific induction of circular RNA during human fetal development. Genome Biol. 2015;16(1):1-26.

13. Chen LL. The biogenesis and emerging roles of circular RNAs. Nat Rev Mol Cell Biol. 2016:17(4):205-11.

14. You X, Vlatkovic I, Babic A, et al. Neural circular RNAs are derived from synaptic genes and regulated by development and plasticity. Nat Neurosci. 2015;18(4):603-10.

15. Nagpal JK, Dasgupta S, Jadallah S, et al. Profiling the expression pattern of GPI transamidase complex subunits in human cancer. Mod Pathol. 2008; 21(8):979-91.

16. Mendell JT, ap Rhys CM, Dietz HC. Separable roles for rent1/hUpf1 in altered splicing and decay of nonsense transcripts. Science. 2002;298(5592):419-22.

17. Kaluza J, Harris HR, Linden A. Alcohol Consumption and Risk of Chronic Obstructive Pulmonary Disease: A Prospective Cohort Study of Men. Am J Epidemiol. 2019:188(5):907-16.

18. Zhang ZJ. The handbook of behavioral medicine [M/CD]. Beijing: China Medical Electronic Audio and Video Publishing House; 2005.

19. Liang J, Wu X, Sun S, et al. Circular RNA expression profile analysis of severe acne by RNA-Seq and bioinformatics. J Eur Acad Dermatol Venereol. 2018; 32(11):1986-92

20. Geng HH, Li R, Su YM, et al. The circular RNA Cdr1as promotes myocardial infarction by mediating the regulation of miR-7a on its target genes expression. PLoS One. 2016;11(3):e0151753.

21. Shan K, Liu C, Liu BH, et al. Circular noncoding RNA HIPK3 mediates retinal vascular dysfunction in diabetes mellitus. Circulation. 2017;136(17):1629-42.

22. Fritz J, Strohmaier S, Nagel G, et al. A strong interaction between age and overweight/obesity on the risk of coronary heart disease in the context of metabolic mediation. Epidemiology. 2016;27(3):e13.

23. Chen Y, Copeland WK, Vedanthan R, et al. Association between body mass index and cardiovascular disease mortality in east Asians and south Asians: pooled analysis of prospective data from the Asia cohort consortium. Bmj. 2013:347:f5446.

24. Loehr LR, Rosamond WD, Poole C, et al. Association of multiple anthropometrics of overweight and obesity with incident heart failure: the atherosclerosis risk in communities study. Circ Heart Fail. 2009;2(1):18-24. 
25. Wang W, Dai LX, Zhang S, et al. Regulation of epidermal growth factor receptor signaling by plasmid-based MicroRNA-7 inhibits human malignant gliomas growth and metastasis in vivo. Neoplasma. 2013;60(3):274-83.

26. Bazan HA, Hatfield SA, Brug A, et al. Carotid Plaque Rupture Is Accompanied by an Increase in the Ratio of Serum circR-284 to miR-221 Levels. Circ Cardiovasc Genet. 2017;10(4). https://doi.org/10.1161/CIRCGENETICS.117.001 720 .

27. Takahashi T, Hiasa Y, Ohara Y, et al. Relationship of admission neutrophil count to microvascular injury, left ventricular dilation, and long-term outcome in patients treated with primary angioplasty for acute myocardial infarction. Circ J. 2008:72(6):867-72.

28. Crea F, Biasucci LM, Buffon A, et al. Role of inflammation in the pathogenesis of unstable coronary artery disease. Am J Cardiol. 1997; 80(5A):10E-6E.

29. Messner B, Bernhard D. Smoking and cardiovascular disease: mechanisms of endothelial dysfunction and early atherogenesis. Arterioscler Thromb Vasc Biol. 2014;34(3):509-15.

30. King CC, Piper ME, Gepner AD, et al. Longitudinal impact of smoking and smoking cessation on inflammatory markers of cardiovascular disease risk. Arterioscler Thromb Vasc Biol. 2017:37(2):374-9.

\section{Publisher's Note}

Springer Nature remains neutral with regard to jurisdictional claims in published maps and institutional affiliations.

Ready to submit your research? Choose BMC and benefit from:

- fast, convenient online submission

- thorough peer review by experienced researchers in your field

- rapid publication on acceptance

- support for research data, including large and complex data types

- gold Open Access which fosters wider collaboration and increased citations

- maximum visibility for your research: over $100 \mathrm{M}$ website views per year

At BMC, research is always in progress.

Learn more biomedcentral.com/submissions 